
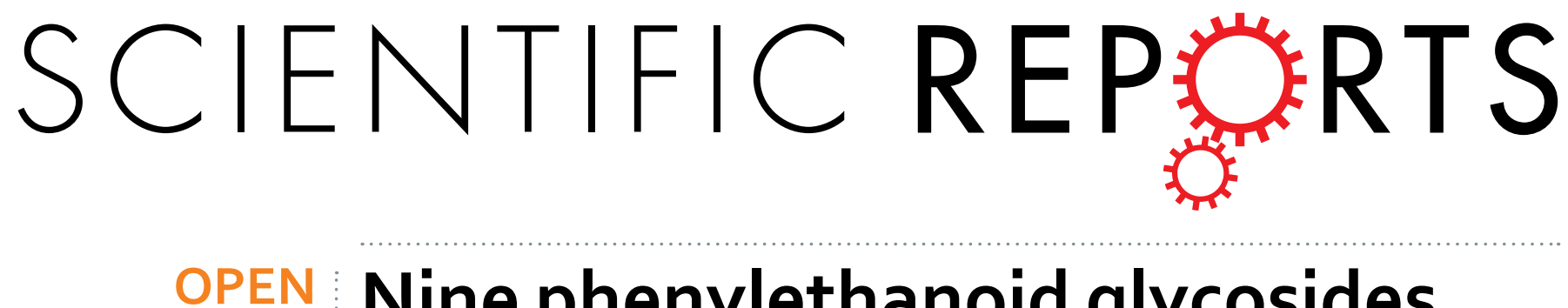

Nine phenylethanoid glycosides from Magnolia officinalis var. biloba fruits and their protective

Received: 08 November 2016

Accepted: 23 February 2017

Published: 28 March 2017 effects against free radical-induced oxidative damage

Lanlan $\mathrm{Ge}^{1}$, Wenhui Zhang ${ }^{1}$, Gao Zhou ${ }^{1}$, Bingxin $\mathrm{Ma}^{1}$, Qigui Mo ${ }^{1}$, Yuxin Chen ${ }^{1}$ \& Youwei Wang ${ }^{1,2}$

To systematically study the chemical constituents in Magnolia officinalis var. biloba fruits, nine phenylethanoid glycosides were isolated by solvent extraction, silica gel, and preparative highperformance liquid chromatography (HPLC). Their structures were elucidated by $1 D$ and 2D NMR analyses, including COSY, HMOC and HMBC correlations, and HPLC analysis of sugar residue. Nine phenylethanoid glycosides, namely, magnoloside $I_{a}(1)$, magnoloside $I_{c}(2)$, crassifolioside (3), magnoloside $\mathrm{I}_{\mathrm{b}}(4)$, magnoloside $\mathrm{III}_{\mathrm{a}}(5)$, magnoloside $\mathrm{IV}_{\mathrm{a}}(6)$, magnoloside $\mathrm{II}_{\mathrm{a}}(7)$, magnoloside $\mathrm{I}_{\mathrm{b}}(8)$ and magnoloside $V_{a}(9)$, were first isolated from the $n$-butanol fraction of Magnolia officinalis var. biloba fruits alcohol extract. Free radical scavenging activities of the nine phenylethanoid glycosides were assessed using the DPPH, ABTS, and superoxide anion radical scavenging assays. Simultaneously, protective effects of all compounds against free radical-induced oxidative damage were evaluated by two different kinds of mitochondrial damage model. The protective effects were assessed by mitochondrial swelling, the formations of malondialdehyde (MDA) and lipid hydroperoxide (LOOH), the activities of catalase (CAT), glutathione reductase (GR) and superoxide dismutase (SOD). All phenylethanoid glycosides showed significant protective effects.

Magnolia bark extracts are derived from Magnolia officinalis Rehd. et Wils. (M. officinalis) or Magnolia officinalis Rehd. et Wils. var. biloba Rehd. et Wils. (M. officinalis var. biloba). They have been used as traditional Chinese medicine (TCM) for treating abdominal distention, nausea, vomiting, dyspepsia, cough, and asthma ${ }^{1}$. Modern pharmacological research showed that magnolia bark extracts have effective antibacterial ${ }^{2}$, antioxidant ${ }^{3,4}$, anti-inflammatory ${ }^{5,6}$, anti-anxiety ${ }^{7}$, anti-gastric ulcer ${ }^{8}$, antitumor ${ }^{9}$, neuroprotective ${ }^{10}$, and cardiovascular protection $^{11}$ activities. The bioactive components of magnolia bark extracts include mainly neolignans, lignans, sesquiterpenes, alkaloids, and phenylethanoid glycosides ${ }^{12-16}$. Recently, magnolia bark extracts have been recognized as a food additive worldwide.

In 2002, the Ministry of Health of the People's Republic of China passed the notice to further standardize the management of health food raw materials, including $M$. officinalis and M. officinalis var. biloba barks, into healthy food materials ${ }^{17}$. In 2008, magnolia bark extracts achieved GRAS status in the United States and were allowed to be added as an ingredient to chewing gums and mints sold in the United States; In 2009, magnolia bark extracts were approved as a new ingredient of food resource by the United Kingdom and European Union; In 2010, the United Kingdom authorities officially approved and specified that the maximum addition amount of magnolia bark extracts in gum and mints is $0.2 \%{ }^{18,19}$. Michael et al. also confirmed that compressed mints and chewing gum containing bark extracts are effective against bacteria responsible for oral malodor ${ }^{20}$. These policies marked the transformation of magnolia bark extracts from TCM to food and dramatically expanded their application. China is the only country to cultivate M. officinalis and M. officinalis var. biloba (the plant area of M. officinalis var. biloba

${ }^{1}$ Institute of TCM and Natural Products, School of Pharmaceutical Sciences, Wuhan University, Wuhan 430071, P. R. China. ${ }^{2}$ MOE Key Laboratory of Combinatorial Biosynthesis and Drug Discovery, Wuhan University, Wuhan 430072, P. R. China. Correspondence and requests for materials should be addressed toY.W. (email: wyw@whu.edu.cn) 


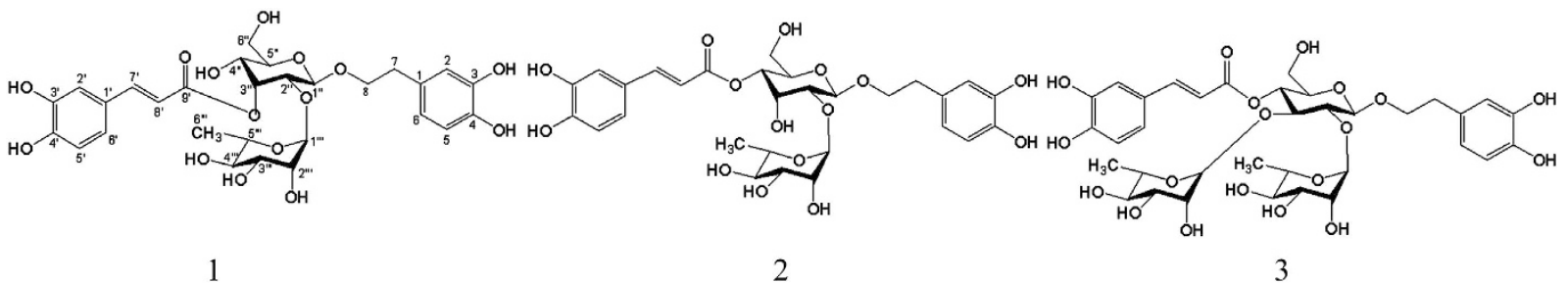

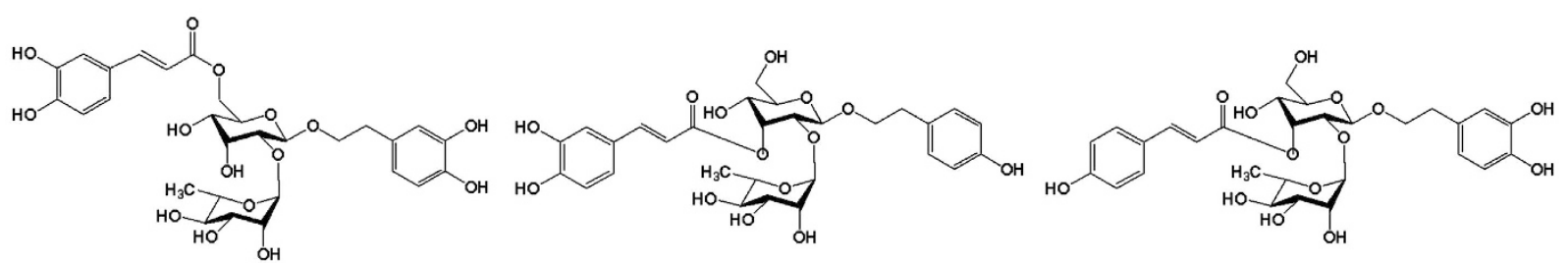

4

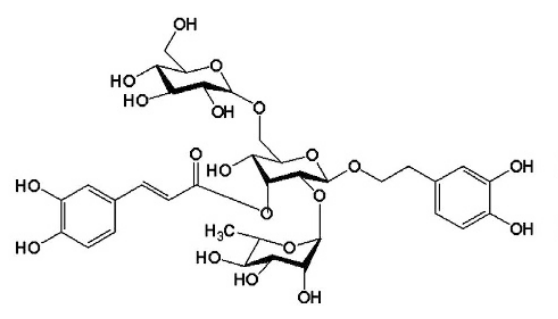

7
5

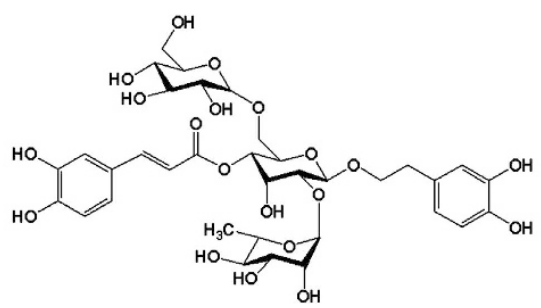

8

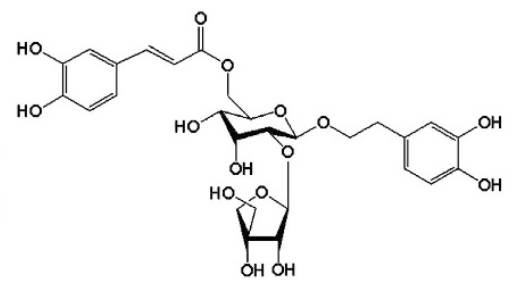

9

Figure 1. Chemical structure of all identified phenylethanoid glycosides in M. officinalis var. biloba fruits.

is wider than that of M. officinalis $)^{21}$, which are obtained largely by cutting down trees and peeling off their barks. However, this destructive utilization pattern is unsuitable for ecological and environmental protection. Therefore, a sustainable utilization pattern must be established to change this situation. M. officinalis and M. officinalis var. biloba fruits, which are nutritious reproductive organs of these plants, can be harvested every year. However, whether their fruits can be used as an alternative food resource remains unknown.

We had already reported that phenylethanoid glycosides were isolated from $M$. officinalis var. biloba fruits in $2015^{22}$. Phenylethanoid glycosides, which exist mostly in Orobanchaceae plants ${ }^{23,24}$, have been demonstrated to possess diverse biological activities, such as antioxidant, anti-inflammatory, antibacterial, antiviral, antitumor, neuroprotective, and immunomodulatory effects ${ }^{25-31}$. However, whether the phenylethanoid glycosides isolated from M. officinalis var. biloba fruits have the same effects as those isolated from Orobanchaceae plants needs to be elucidated. Moreover, we have not validated whether the analogous components from their fruits can be associated with the traditional functions of magnolia bark extracts. Therefore, more studies are needed to further understand the function of phenylethanoid glycosides.

In view of the more abundant resources of M. officinalis var. biloba, we focused on the $n$-butanol fraction of the alcohol extract from M. officinalis var. biloba fruits and isolated nine phenylethanoid glycosides (Fig. 1). The isolation and structural elucidation of these nine phenylethanoid glycosides, as well as their free radical scavenging activities, are reported in this paper. Subsequently, their protective effects against free radical-induced oxidative damage in two different kinds of mitochondrial damage model were also evaluated.

\section{Results and Disscussion}

Structural identification of phenylethanoid glycosides. Compounds 1-9 were identified as phenylethanoid glycosides. Their structures were characterized by the phenylpropionyl group and benzene ethanol group through ester linkage and glycoside bond connected to the central sugar, respectively.

Compound 2 was isolated as a light yellow amorphous powder. Its molecular formula was $\mathrm{C}_{29} \mathrm{H}_{36} \mathrm{O}_{15}$, which was deduced from the molecular ion peak at $m / z 623.1999[\mathrm{M}-\mathrm{H}]^{-}\left(\right.$calcd. $\left.\mathrm{C}_{29} \mathrm{H}_{35} \mathrm{O}_{15}, 623.1981\right)$ by HR-ESI-MS and supported by the ${ }^{13} \mathrm{C}$ NMR spectral data. The IR spectrum of 2 displaced the characteristic absorption bands for the hydroxyl group $\left(3410 \mathrm{~cm}^{-1}\right)$, conjugated carbonyl group $\left(1687 \mathrm{~cm}^{-1}\right)$, aromatic rings $(1604$ and $\left.1516 \mathrm{~cm}^{-1}\right)$, and glycosidic group $\left(816 \mathrm{~cm}^{-1}\right)$. The UV spectrum obtained the maximum absorption at 206, 291, and $330 \mathrm{~nm}$.

The ${ }^{1} \mathrm{H}-\mathrm{NMR}$ and ${ }^{13} \mathrm{C}$ NMR spectra of $\mathbf{2}$ (Tables 1,2 and supplementary information) exhibited characteristic signals arising from the trans-caffeoyl group and 3,4-dihydroxy phenethyl alcohol group showing two sets of ABX-type signals caused by aromatic protons at $\delta_{\mathrm{H}} 7.08(1 \mathrm{H}, \mathrm{d}, J=2.0 \mathrm{~Hz}), 6.97(1 \mathrm{H}, \mathrm{dd}, J=2.0,8.0 \mathrm{~Hz}), 6.80$ $(1 \mathrm{H}, \mathrm{d}, J=8.0 \mathrm{~Hz})$ and $\delta_{\mathrm{H}} 6.73(1 \mathrm{H}, \mathrm{d}, J=2.0 \mathrm{~Hz}), 6.71(1 \mathrm{H}, \mathrm{d}, J=8.0 \mathrm{~Hz}), 6.58(1 \mathrm{H}, \mathrm{dd}, J=2.0,8.0 \mathrm{~Hz})$, a pair 


\begin{tabular}{|c|c|c|c|}
\hline \multirow[b]{2}{*}{ Position } & \multicolumn{3}{|c|}{${ }^{1} \mathrm{H}-\mathrm{NMR}$} \\
\hline & 2 & 8 & 3 \\
\hline & \multicolumn{3}{|c|}{ Aglycone } \\
\hline 1 & - & - & - \\
\hline 2 & $6.73(1 \mathrm{H}, \mathrm{d}, 2.0)$ & $6.74(1 \mathrm{H}, \mathrm{d}, 2.0)$ & $6.71(1 \mathrm{H}, \mathrm{d}, 2.0)$ \\
\hline 3 & - & - & - \\
\hline 4 & - & - & - \\
\hline 5 & $6.71(1 \mathrm{H}, \mathrm{d}, 8.0)$ & $6.71(1 \mathrm{H}, \mathrm{d}, 8.0)$ & $6.69(1 \mathrm{H}, \mathrm{d}, 8.0)$ \\
\hline 6 & $6.58(1 \mathrm{H}, \mathrm{dd}, 2.0,8.0)$ & $6.60(1 \mathrm{H}, \mathrm{dd}, 2.0,8.0)$ & $6.58(1 \mathrm{H}, \mathrm{dd}, 2.0,8.0)$ \\
\hline 7 & $2.80(2 \mathrm{H}, \mathrm{t}, 7.4)$ & $2.80(2 \mathrm{H}, \mathrm{t}, 7.4)$ & $2.84(2 \mathrm{H}, \mathrm{t}, 8.0)$ \\
\hline \multirow[t]{2}{*}{8} & $3.94(1 \mathrm{H}, \mathrm{m}) ; 3.77(1 \mathrm{H}, \mathrm{m})$ & $3.65(1 \mathrm{H}, \mathrm{m}) ; 3.76(1 \mathrm{H}, \mathrm{m})$ & $3.69(1 \mathrm{H}, \mathrm{m}) ; 3.91(1 \mathrm{H}, \mathrm{m})$ \\
\hline & \multicolumn{3}{|c|}{ Caffeoyl } \\
\hline $1^{\prime}$ & - & - & - \\
\hline $2^{\prime}$ & $7.08(1 \mathrm{H}, \mathrm{d}, 2.0)$ & $7.09(1 \mathrm{H}, \mathrm{d}, 2.0)$ & $7.07(1 \mathrm{H}, \mathrm{d}, 2.0)$ \\
\hline $3^{\prime}$ & - & - & - \\
\hline $4^{\prime}$ & - & - & - \\
\hline $5^{\prime}$ & $6.80(1 \mathrm{H}, \mathrm{d}, 8.0)$ & $6.81(1 \mathrm{H}, \mathrm{d}, 8.0)$ & $6.80(1 \mathrm{H}, \mathrm{d}, 8.0)$ \\
\hline $6^{\prime}$ & $6.97(1 \mathrm{H}, \mathrm{dd}, 2.0,8.0)$ & $6.97(1 \mathrm{H}, \mathrm{dd}, 2.0,8.0)$ & $6.97(1 \mathrm{H}, \mathrm{dd}, 2.0,8.0)$ \\
\hline $7^{\prime}$ & $7.63(1 \mathrm{H}, \mathrm{d}, 15.9)$ & $7.63(1 \mathrm{H}, \mathrm{d}, 15.9)$ & $7.60(1 \mathrm{H}, \mathrm{d}, 15.9)$ \\
\hline $8^{\prime}$ & $6.33(1 \mathrm{H}, \mathrm{d}, 15.9)$ & $6.33(1 \mathrm{H}, \mathrm{d}, 15.9)$ & $6.30(1 \mathrm{H}, \mathrm{d}, 15.9)$ \\
\hline \multirow[t]{2}{*}{$9^{\prime}$} & - & - & - \\
\hline & \multicolumn{2}{|c|}{ Allose } & Glucose \\
\hline $1^{\prime \prime}$ & $4.85(1 \mathrm{H}, \mathrm{d}, 8.0)$ & $4.85(1 \mathrm{H}, \mathrm{d}, 8.0)$ & $4.51(1 \mathrm{H}, \mathrm{d}, 7.7)$ \\
\hline $2^{\prime \prime}$ & $3.47(1 \mathrm{H}, \mathrm{m})$ & $3.46(1 \mathrm{H}, \mathrm{m})$ & $3.97(1 \mathrm{H}, \mathrm{m})$ \\
\hline $3^{\prime \prime}$ & $4.51(1 \mathrm{H}, \mathrm{s})$ & $4.51(1 \mathrm{H}, \mathrm{s})$ & $3.57(1 \mathrm{H}, \mathrm{m})$ \\
\hline $4^{\prime \prime}$ & $4.84(1 \mathrm{H}, \mathrm{m})$ & $4.92(1 \mathrm{H}, \mathrm{m})$ & $4.96(1 \mathrm{H}, \mathrm{m})$ \\
\hline $5^{\prime \prime}$ & $3.60(1 \mathrm{H}, \mathrm{m})$ & $3.22(1 \mathrm{H}, \mathrm{m})$ & $3.51(1 \mathrm{H}, \mathrm{m})$ \\
\hline \multirow[t]{2}{*}{$6^{\prime \prime}$} & $3.62(1 \mathrm{H}, \mathrm{m}) ; 3.79(1 \mathrm{H}, \mathrm{m})$ & $3.67(1 \mathrm{H}, \mathrm{m}) ; 4.01(1 \mathrm{H}, \mathrm{m})$ & $3.52(1 \mathrm{H}, \mathrm{m}) ; 3.63(1 \mathrm{H}, \mathrm{m})$ \\
\hline & \multicolumn{3}{|c|}{ 2"-Rhamnose } \\
\hline $1^{\prime \prime \prime}$ & $4.90(1 \mathrm{H}, \mathrm{s})$ & $4.89(1 \mathrm{H}, \mathrm{s})$ & $4.96(1 \mathrm{H}, \mathrm{s})$ \\
\hline $2^{\prime \prime \prime}$ & $3.68(1 \mathrm{H}, \mathrm{m})$ & $4.02(1 \mathrm{H}, \mathrm{m})$ & $3.46(1 \mathrm{H}, \mathrm{m})$ \\
\hline $3^{\prime \prime \prime}$ & $3.61(1 \mathrm{H}, \mathrm{m})$ & $4.24(1 \mathrm{H}, \mathrm{m})$ & $3.84(1 \mathrm{H}, \mathrm{m})$ \\
\hline $4^{\prime \prime \prime}$ & $3.36(1 \mathrm{H}, \mathrm{m})$ & $3.93(1 \mathrm{H}, \mathrm{m})$ & $3.32(1 \mathrm{H}, \mathrm{m})$ \\
\hline $5^{\prime \prime \prime}$ & $4.04(1 \mathrm{H}, \mathrm{m})$ & $3.67(1 \mathrm{H}, \mathrm{m})$ & $4.05(1 \mathrm{H}, \mathrm{m})$ \\
\hline \multirow[t]{2}{*}{$6^{\prime \prime \prime}$} & $1.29(3 \mathrm{H}, \mathrm{d}, 6.0)$ & $1.30(3 \mathrm{H}, \mathrm{d}, 6.0)$ & $1.14(3 \mathrm{H}, \mathrm{d}, 6.0)$ \\
\hline & & 6"-Glucose & 3"-Rhamnose \\
\hline $1^{\prime \prime \prime \prime \prime}$ & & $4.30(1 \mathrm{H}, \mathrm{d}, 7.7)$ & $5.01(1 \mathrm{H}, \mathrm{s})$ \\
\hline $2^{\prime \prime \prime \prime \prime}$ & & $3.60(1 \mathrm{H}, \mathrm{m})$ & $3.46(1 \mathrm{H}, \mathrm{m})$ \\
\hline $3^{\prime \prime \prime \prime}$ & & $3.25(1 \mathrm{H}, \mathrm{m})$ & $3.84(1 \mathrm{H}, \mathrm{m})$ \\
\hline $4^{\prime \prime \prime \prime \prime}$ & & $3.32(1 \mathrm{H}, \mathrm{m})$ & $3.32(1 \mathrm{H}, \mathrm{m})$ \\
\hline $5^{\prime \prime \prime \prime \prime}$ & & $3.35(1 \mathrm{H}, \mathrm{m})$ & $4.05(1 \mathrm{H}, \mathrm{m})$ \\
\hline $6^{\prime \prime \prime \prime \prime}$ & & $3.64(1 \mathrm{H}, \mathrm{m}) ; 3.86(1 \mathrm{H}, \mathrm{m})$ & $1.29(3 \mathrm{H}, \mathrm{d}, 6.0)$ \\
\hline
\end{tabular}

Table 1. ${ }^{1} \mathrm{H}-\mathrm{NMR}\left(\mathrm{CD}_{3} \mathrm{OD}, 400 \mathrm{MHz}\right)$ data of compounds 2,8 , and $3\left(\delta_{\mathrm{H}}, \mathrm{dppm}\right)$.

of trans-olefinic proton signals at $\delta_{\mathrm{H}} 7.63(1 \mathrm{H}, \mathrm{d}, J=15.9 \mathrm{~Hz}), 6.33(1 \mathrm{H}, \mathrm{d}, J=15.9 \mathrm{~Hz})$, and benzylic methylene proton signal at $\delta_{\mathrm{H}} 2.80(2 \mathrm{H}, \mathrm{t}, J=7.4 \mathrm{~Hz})$, which were assigned to $\delta_{\mathrm{C}} 115.30\left(\mathrm{C}-2^{\prime}\right), 123.20\left(\mathrm{C}-6^{\prime}\right), 116.45\left(\mathrm{C}-5^{\prime}\right)$, $117.25(\mathrm{C}-2), 116.59(\mathrm{C}-5), 121.41(\mathrm{C}-6), 147.72\left(\mathrm{C}-7^{\prime}\right), 114.71\left(\mathrm{C}-8^{\prime}\right)$, and $36.73(\mathrm{C}-7)$, respectively, via HMQC correlation. Additionally, two anomeric proton resonances appeared at $\delta_{\mathrm{H}} 4.85(1 \mathrm{H}, \mathrm{d}, J=8.0 \mathrm{~Hz})$ and $4.90(1 \mathrm{H}$, s), which correlated with signals at $\delta_{\mathrm{C}} 100.47\left(\mathrm{C}-1^{\prime \prime}\right)$ and $97.91\left(\mathrm{C}-1^{\prime \prime \prime}\right)$ in the HMQC spectrum, respectively. The ${ }^{1} \mathrm{H}$-NMR spectrum also showed the presence of a methyl group at $\delta_{\mathrm{H}} 1.29(3 \mathrm{H}, \mathrm{d}, J=6.0 \mathrm{~Hz})$, which indicated the presence of rhamnose in the compound. A series of signals in ${ }^{1} \mathrm{H}-\mathrm{NMR}\left(\mathrm{CD}_{3} \mathrm{OD}, 400 \mathrm{MHz}\right)$ at $\delta_{\mathrm{H}} 4.85(1 \mathrm{H}, \mathrm{d}$, $J=8.0 \mathrm{~Hz}), 3.47(1 \mathrm{H}, \mathrm{m}), 4.51(1 \mathrm{H}, \mathrm{s}), 4.84(1 \mathrm{H}, \mathrm{m}), 3.60(1 \mathrm{H}, \mathrm{m}), 3.62(1 \mathrm{H}, \mathrm{m})$, and $3.79(1 \mathrm{H}, \mathrm{m})$ and ${ }^{13} \mathrm{C} \mathrm{NMR}$ $\left(\mathrm{CD}_{3} \mathrm{OD}, 100 \mathrm{MHz}\right)$ at $\delta_{\mathrm{C}} 100.47\left(\mathrm{C}-1^{\prime \prime}\right), 73.19\left(\mathrm{C}-2^{\prime \prime}\right), 66.45\left(\mathrm{C}-3^{\prime \prime}\right), 70.57\left(\mathrm{C}-4^{\prime \prime}\right), 74.53\left(\mathrm{C}-5^{\prime \prime}\right)$, and $62.37\left(\mathrm{C}-6^{\prime \prime}\right.$ ) indicated a rare $\beta$-allopyranosyl unit. Moreover, this unit was confirmed by comparing the NMR features of those of magnolosides A, B, C, D, and $\mathrm{E}^{16,32,33}$ isolated from $M$. officinalis barks or M. obovata barks, which also contained allopyranose. Acid hydrolysate of compound 2 contained monosaccharide components, which were identified as D-allose and L-rhamnose by HPLC analysis. In the HMBC relationship of 2 (Fig. 2), the correlations between $\mathrm{H}-1^{\prime \prime}\left(\delta_{\mathrm{H}} 4.85\right)$ and $\mathrm{C}-8$ (72.30) showed that the phenylethanol moiety was linked to C-1" (All-C- $\left.1^{\prime \prime}\right)$; the cross peak between $\mathrm{H}-4^{\prime \prime}\left(\delta_{\mathrm{H}} 4.84\right)$ and C-9' (168.14) showed that the trans-caffeoyl moiety was linked to C- $4^{\prime \prime}$ 


\begin{tabular}{|c|c|c|c|}
\hline \multirow[b]{2}{*}{ Position } & \multicolumn{3}{|c|}{${ }^{13} \mathrm{C}-\mathrm{NMR}$} \\
\hline & 2 & 8 & 3 \\
\hline \multicolumn{4}{|c|}{ Aglycone } \\
\hline 1 & 131.68 & 131.67 & 130.70 \\
\hline 2 & 117.25 & 116.63 & 115.87 \\
\hline 3 & 146.82 & 146.80 & 146.32 \\
\hline 4 & 144.61 & 144.59 & 144.17 \\
\hline 5 & 116.59 & 117.31 & 116.64 \\
\hline 6 & 121.41 & 121.51 & 120.78 \\
\hline 7 & 36.73 & 36.72 & 36.04 \\
\hline 8 & 72.30 & 72.45 & 71.86 \\
\hline \multicolumn{4}{|c|}{ Caffeoyl } \\
\hline $1^{\prime}$ & 127.70 & 127.68 & 127.17 \\
\hline $2^{\prime}$ & 115.30 & 115.37 & 114.74 \\
\hline $3^{\prime}$ & 146.01 & 145.97 & 145.61 \\
\hline $4^{\prime}$ & 149.75 & 149.77 & 149.26 \\
\hline $5^{\prime}$ & 116.45 & 116.53 & 116.04 \\
\hline $6^{\prime}$ & 123.20 & 123.33 & 122.70 \\
\hline $7^{\prime}$ & 147.72 & 147.96 & 147.38 \\
\hline $8^{\prime}$ & 114.71 & 114.66 & 114.47 \\
\hline $9^{\prime}$ & 168.14 & 168.23 & 167.91 \\
\hline \multicolumn{3}{|c|}{ Allose } & Glucose \\
\hline $1^{\prime \prime}$ & 100.47 & 100.50 & 102.46 \\
\hline $2^{\prime \prime}$ & 73.19 & 73.97 & 82.68 \\
\hline $3^{\prime \prime}$ & 66.45 & 66.42 & 80.43 \\
\hline $4^{\prime \prime}$ & 70.57 & 70.64 & 69.83 \\
\hline $5^{\prime \prime}$ & 74.53 & 75.03 & 75.50 \\
\hline \multirow[t]{2}{*}{$6^{\prime \prime}$} & 62.37 & 69.62 & 61.88 \\
\hline & \multicolumn{3}{|c|}{$2^{\prime \prime}$-Rhamnose } \\
\hline $1^{\prime \prime \prime}$ & 97.91 & 97.91 & 103.07 \\
\hline $2^{\prime \prime \prime}$ & 72.37 & 72.19 & 72.01 \\
\hline $3^{\prime \prime \prime}$ & 72.22 & 72.25 & 71.71 \\
\hline $4^{\prime \prime \prime}$ & 73.99 & 72.36 & 73.33 \\
\hline $5^{\prime \prime \prime}$ & 69.99 & 70.01 & 70.71 \\
\hline \multirow[t]{2}{*}{$6^{\prime \prime \prime}$} & 18.04 & 18.09 & 17.91 \\
\hline & & $6^{\prime \prime}$-Glucose & $3^{\prime \prime}$-Rhamnose \\
\hline $1^{\prime \prime \prime \prime}$ & & 104.92 & 102.32 \\
\hline $2^{\prime \prime \prime \prime}$ & & 74.41 & 71.80 \\
\hline $3^{\prime \prime \prime \prime}$ & & 77.78 & 71.49 \\
\hline $4^{\prime \prime \prime \prime}$ & & 71.48 & 73.18 \\
\hline $5^{\prime \prime \prime \prime}$ & & 77.90 & 70.43 \\
\hline $6^{\prime \prime \prime \prime}$ & & 62.67 & 17.69 \\
\hline
\end{tabular}

Table 2. ${ }^{13} \mathrm{C}-\mathrm{NMR}\left(\mathrm{CD}_{3} \mathrm{OD}, 100 \mathrm{MHz}\right)$ data of compounds 2,8 , and $3\left(\delta_{\mathrm{C}}, \mathrm{dppm}\right)$.

(All-C- $\left.4^{\prime \prime}\right)$; and the long-range correlations between $\mathrm{H}-1^{\prime \prime \prime}\left(\delta_{\mathrm{H}} 4.90\right)$ and C-2" $(73.19)($ All-C-2" $)$ showed that the rhamnose moiety was linked to C- $2^{\prime \prime}$ (All-C-2").

In light of all the above considerations, the structure of 2 was revealed as 2-(3, 4-dihydroxyphenyl) -ethyl-O-a-L-rhamnopyranosyl-( $1 \rightarrow 2)$-(4-O-trans-caffeoyl)- $\beta$-D-allopyranoside.

Compound 3 was obtained as a canary yellow amorphous powder. The HR-ESI-MS data showed an accurate $[\mathrm{M}-\mathrm{H}]^{-}$ion at $m / z 769.2668$ (calcd. for $\mathrm{C}_{35} \mathrm{H}_{45} \mathrm{O}_{19}, 769.2654$ ), in accordance with the empirical molecular formula of $\mathrm{C}_{35} \mathrm{H}_{46} \mathrm{O}_{19}$. The IR spectrum of 3 displaced characteristic absorption bands for the hydroxyl group $\left(3402 \mathrm{~cm}^{-1}\right)$, conjugated carbonyl group $\left(1699 \mathrm{~cm}^{-1}\right)$, aromatic rings $\left(1603\right.$ and $\left.1517 \mathrm{~cm}^{-1}\right)$, and glycosidic group $\left(814 \mathrm{~cm}^{-1}\right)$. The UV spectrum obtained the maximum absorption at 206, 280, and $330 \mathrm{~nm}$.

The ${ }^{1} \mathrm{H}-\mathrm{NMR}$ and ${ }^{13} \mathrm{C}$ NMR spectra of 3 (Tables 1, 2 and supplementary information) exhibited the same characteristic signals to prove the existence of the trans-caffeoyl group and 3,4-dihydroxy phenethyl alcohol group as compound 2. By contrast, three sugar anomeric protons were observed at $\delta_{\mathrm{H}} 5.01(1 \mathrm{H}, \mathrm{s}), 4.96(1 \mathrm{H}, \mathrm{s})$, and 4.51 $(1 \mathrm{H}, \mathrm{d}, J=7.7 \mathrm{~Hz})$ and resonated at $\delta_{\mathrm{C}} 102.32\left(\mathrm{C}-1^{\prime \prime \prime \prime}\right), 103.07\left(\mathrm{C}-1^{\prime \prime \prime}\right)$, and $102.46\left(\mathrm{C}-1^{\prime}\right)$ in ${ }^{13} \mathrm{C}$ NMR, respectively. They also could be supported in the HMQC spectrum. Two methyl groups at $\delta_{\mathrm{H}} 1.14(3 \mathrm{H}, \mathrm{d}, J=6.0 \mathrm{~Hz})$ and 1.29 $(3 \mathrm{H}, \mathrm{d}, J=6.0 \mathrm{~Hz})$, as well as $\delta_{\mathrm{C}} 17.91\left(\mathrm{C}-6^{\prime \prime \prime}\right)$ and $17.69\left(\mathrm{C}-6^{\prime \prime \prime}\right)$, indicated that compound 3 may contain two 


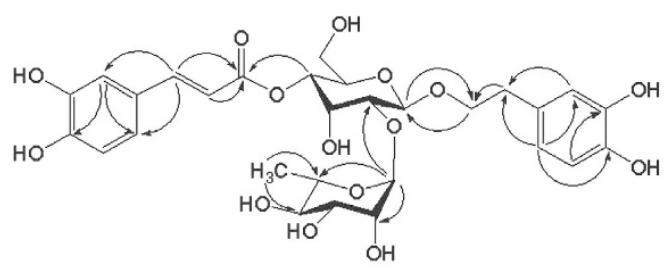

compound 2

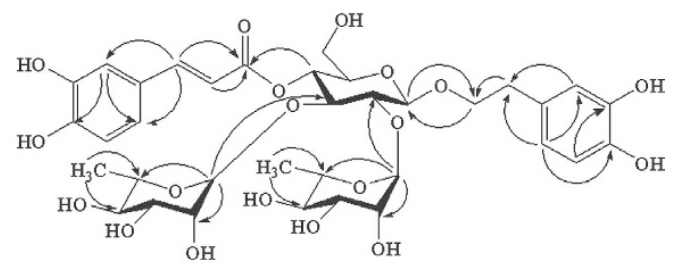

compound 3

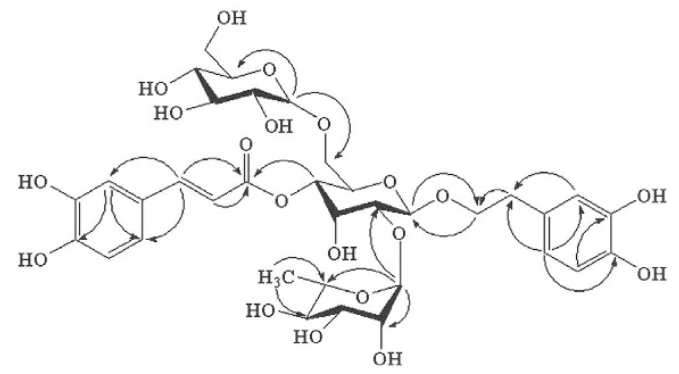

compound $\mathbf{8}$

Figure 2. Key HMBC correlations $(\mathrm{H} \rightarrow \mathrm{C})$ of compounds 2,8 , and 3 .

rhamnoses. A series of signals in ${ }^{1} \mathrm{H}-\mathrm{NMR}\left(\mathrm{CD}_{3} \mathrm{OD}, 400 \mathrm{MHz}\right)$ at $\delta_{\mathrm{H}} 4.51(1 \mathrm{H}, \mathrm{d}, J=7.7 \mathrm{~Hz}), 3.97(1 \mathrm{H}, \mathrm{m}), 3.57$ $(1 \mathrm{H}, \mathrm{m}), 4.96(1 \mathrm{H}, \mathrm{m}), 3.51(1 \mathrm{H}, \mathrm{m}), 3.52(1 \mathrm{H}, \mathrm{m})$, and $3.63(1 \mathrm{H}, \mathrm{m})$ and in ${ }^{13} \mathrm{C} \mathrm{NMR}\left(\mathrm{CD}_{3} \mathrm{OD}, 100 \mathrm{MHz}\right)$ at $\delta_{\mathrm{C}}$ $102.46\left(\mathrm{C}-1^{\prime \prime}\right), 82.68\left(\mathrm{C}-2^{\prime \prime}\right), 80.43\left(\mathrm{C}-3^{\prime \prime}\right), 69.83\left(\mathrm{C}-4^{\prime \prime}\right), 75.50\left(\mathrm{C}-5^{\prime \prime}\right)$, and $61.88\left(\mathrm{C}-6^{\prime \prime}\right)$ showed that the central sugar was $\beta$-glucose. The acid hydrolysate of compound 3 further confirmed the existence of D-glucose and L-rhamnose, which were identified by HPLC analysis. In the HMBC relationship of 3 (Fig. 2), the correlations between $\mathrm{H}-1^{\prime \prime}\left(\delta_{\mathrm{H}} 4.51\right)$ and $\mathrm{C}-8\left(\delta_{\mathrm{C}} 71.86\right)$ showed that the phenylethanol moiety was linked to C- $1^{\prime \prime}\left(\mathrm{Glc}-\mathrm{C}-1^{\prime \prime}\right)$; the cross peak between $\mathrm{H}-4^{\prime \prime}\left(\delta_{\mathrm{H}} 4.96\right)$ and C-9' $\left(\delta_{\mathrm{C}} 167.91\right)$ showed that the trans-caffeoyl moiety was linked to $\mathrm{C}-4^{\prime \prime}\left(\mathrm{Glc}-\mathrm{C}-4^{\prime \prime}\right)$; and the long-range correlations between $\mathrm{H}-1^{\prime \prime \prime}\left(\delta_{\mathrm{H}} 4.96\right)$ to $\mathrm{C}-2^{\prime \prime}\left(\delta_{\mathrm{C}} 82.68\right)\left(\mathrm{Glc}-\mathrm{C}-2^{\prime \prime}\right)$ and $\mathrm{H}-1^{\prime \prime \prime \prime}$ $\left(\delta_{\mathrm{H}} 5.01\right)$ to $\mathrm{C}-3^{\prime \prime}\left(\delta_{\mathrm{C}} 80.43\right)\left(\mathrm{Glc}-\mathrm{C}-3^{\prime \prime}\right)$ unambiguously showed that the two rhamnose moieties were linked at $\mathrm{C}-2^{\prime \prime}$ and C- $3^{\prime \prime}$, respectively.

All these results confirmed the structure of compound 3 as 2-(3, 4-dihydroxyphenyl)-ethyl-O-a-L-rhamnopyranosyl -( $1 \rightarrow 2)$-[a-L-rhamnopyranosyl-( $1 \rightarrow 3)$ ]-(4-O-trans-caffeoyl)- $\beta$-D-glucopyranoside, named crassifolioside ${ }^{34}$.

Compound 8 was obtained as a light yellow amorphous powder. The negative-ion quasi-molecular ion peak in its HRESI-MS data at $\mathrm{m} / z 785.2520$ [M-H] ${ }^{-}$(calcd. for $\mathrm{C}_{35} \mathrm{H}_{45} \mathrm{O}_{20}, 785.2510$ ) together with the ${ }^{13} \mathrm{C}$ NMR spectral data suggested a molecular formula of $\mathrm{C}_{35} \mathrm{H}_{46} \mathrm{O}_{20}$. The IR spectrum of $\mathbf{8}$ displaced characteristic absorption bands for the hydroxyl group $\left(3234 \mathrm{~cm}^{-1}\right)$, conjugated carbonyl group $\left(1692 \mathrm{~cm}^{-1}\right)$, aromatic rings $(1604$ and $\left.1518 \mathrm{~cm}^{-1}\right)$, and glycosidic group $\left(813 \mathrm{~cm}^{-1}\right)$. The UV spectrum obtained the maximum absorption at 205, 280, and $330 \mathrm{~nm}$.

Comparison of the ${ }^{1} \mathrm{H}-\mathrm{NMR}\left(\mathrm{CD}_{3} \mathrm{OD}, 400 \mathrm{MHz}\right)$ and ${ }^{13} \mathrm{C}$ NMR $\left(\mathrm{CD}_{3} \mathrm{OD}, 100 \mathrm{MHz}\right)$ spectra of 8 (Tables 1,2 and supplementary information) with those of $\mathbf{2}$ suggested that compound $\mathbf{8}$ contained one more sugar group than 2. The extra series of sugar signals in ${ }^{1} \mathrm{H}-\mathrm{NMR}$ at $\delta_{\mathrm{H}} 4.30(1 \mathrm{H}, \mathrm{d}, J=7.7 \mathrm{~Hz}), 3.60(1 \mathrm{H}, \mathrm{m}), 3.25(1 \mathrm{H}, \mathrm{m})$, $3.32(1 \mathrm{H}, \mathrm{m}), 3.35(1 \mathrm{H}, \mathrm{m}), 3.64(1 \mathrm{H}, \mathrm{m})$, and $3.86(1 \mathrm{H}, \mathrm{m})$ and in ${ }^{13} \mathrm{C}$ NMR at $\delta_{\mathrm{C}} 104.92\left(\mathrm{C}-1^{\prime \prime \prime \prime}\right), 74.41\left(\mathrm{C}-2^{\prime \prime \prime \prime}\right)$, $77.78\left(\mathrm{C}-3^{\prime \prime \prime \prime}\right), 71.48\left(\mathrm{C}-4^{\prime \prime \prime \prime}\right), 77.90\left(\mathrm{C}-5^{\prime \prime \prime \prime}\right)$, and $62.67\left(\mathrm{C}-6^{\prime \prime \prime \prime}\right)$ deduced that the extra sugar group was $\beta$-glucose. Acid hydrolysis of 8 further gave $\mathrm{D}$-allose, $\mathrm{D}$-glucose, and L-rhamnose as sugar residues by HPLC analysis. The $7.25 \mathrm{ppm}$ down-field shift of C-6" in the ${ }^{13} \mathrm{C}$ NMR spectrum indicated the attachment of the extra sugar group to $C-6^{\prime \prime}$ of the central sugar. This finding was also supported by the HMBC correlation (Fig. 2) of $\mathrm{H}-1^{\prime \prime \prime \prime}\left(\delta_{\mathrm{H}} 4.30\right)$ and C-6" $\left.\delta_{\mathrm{C}} 69.62\right)$.

Based on the above evidence, compound 8 was established as 2-(3, 4-dihydroxyphenyl)-ethyl-Oa-L-rhamnopyranosyl- $(1 \rightarrow 2)$-[ $\beta$-D-glucopyranosyl- $(1 \rightarrow 6)]$-(4-O-trans-caffeoyl)- $\beta$-D-allopyranoside.

Compounds 5 and $\mathbf{6}$ were isolated as yellow amorphous powder, with the molecular formula of $\mathrm{C}_{29} \mathrm{H}_{36} \mathrm{O}_{14}$, as deduced from the $[\mathrm{M}-\mathrm{H}]^{-}$peak at $\mathrm{m} / z 607.2011$ (calcd. for $\mathrm{C}_{29} \mathrm{H}_{35} \mathrm{O}_{14}, 607.2021$ ) and 607.2009 (calcd. for $\left.\mathrm{C}_{29} \mathrm{H}_{35} \mathrm{O}_{14}, 607.2021\right)$ via HR-ESI-MS and supported by the ${ }^{13} \mathrm{C}$ NMR spectral data. The ${ }^{1} \mathrm{H}-\mathrm{NMR}$ and ${ }^{13} \mathrm{C}$ NMR spectra of 5 (Table 3 and supplementary information) were very similar to those of compound $\mathbf{1}$ (magnoloside A), except for the signals caused by the 4-hydroxyphenylethyl alcohol group \{ortho-coupled $\mathrm{A}_{2} \mathrm{~B}_{2}$-type aromatic protons $\left[\delta_{\mathrm{H}} 7.10(2 \mathrm{H}, \mathrm{d}, J=2.0 \mathrm{~Hz}), 6.71(2 \mathrm{H}, \mathrm{d}, J=8.0 \mathrm{~Hz})\right]$ and two methylenes $\left[\delta_{\mathrm{H}} 2.85(2 \mathrm{H}, \mathrm{t}, J=8.0 \mathrm{~Hz}), 3.67(2 \mathrm{H}\right.$, $\mathrm{m})]\}$, which was also supported by the literature ${ }^{35}$. Comparison of the NMR data of 6 (Table 3 and supplementary information) with those of compound 1 (magnoloside A) revealed that the caffeoyl group of magnoloside A was replaced by the trans-p-coumaroyl group \{ortho-coupled $\mathrm{A}_{2} \mathrm{~B}_{2}$-type aromatic protons $\left[\delta_{\mathrm{H}} 7.51(2 \mathrm{H}, \mathrm{d}, J=8.0 \mathrm{~Hz})\right.$, 


\begin{tabular}{|c|c|c|c|c|}
\hline \multirow[b]{2}{*}{ Position } & \multicolumn{2}{|l|}{5} & \multicolumn{2}{|l|}{6} \\
\hline & $\delta_{\mathrm{H}}$ & $\delta_{\mathrm{C}}$ & $\delta_{\mathrm{H}}$ & $\delta_{\mathrm{C}}$ \\
\hline \multicolumn{5}{|c|}{ Aglycone } \\
\hline 1 & - & 130.80 & - & 131.60 \\
\hline 2 & $7.10(1 \mathrm{H}, \mathrm{d}, 2.0)$ & 131.02 & $6.72(1 \mathrm{H}, \mathrm{d}, 2.0)$ & 117.17 \\
\hline 3 & $6.71(1 \mathrm{H}, \mathrm{d}, 8.0)$ & 116.18 & - & 146.91 \\
\hline 4 & - & 156.74 & - & 144.62 \\
\hline 5 & $6.71(1 \mathrm{H}, \mathrm{d}, 8.0)$ & 116.18 & $6.69(1 \mathrm{H}, \mathrm{d}, 8.0)$ & 116.37 \\
\hline 6 & $7.10(1 \mathrm{H}, \mathrm{d}, 2.0)$ & 131.02 & $6.59(1 \mathrm{H}, \mathrm{dd}, 2.0,8.0)$ & 121.37 \\
\hline 7 & $2.85(2 \mathrm{H}, \mathrm{t}, 8.0)$ & 36.57 & $2.79(2 \mathrm{H}, \mathrm{t}, 8.0)$ & 36.75 \\
\hline \multirow[t]{2}{*}{8} & $3.67(2 \mathrm{H}, \mathrm{m})$ & 72.19 & $3.67(2 \mathrm{H}, \mathrm{m})$ & 72.15 \\
\hline & \multicolumn{2}{|l|}{ Caffeoyl } & \multicolumn{2}{|l|}{ Coumaroyl } \\
\hline $1^{\prime}$ & - & 127.82 & - & 127.24 \\
\hline $2^{\prime}$ & $7.08(1 \mathrm{H}, \mathrm{d}, 2.0)$ & 115.31 & $7.51(1 \mathrm{H}, \mathrm{d}, 8.0)$ & 131.30 \\
\hline $3^{\prime}$ & - & 146.84 & $6.84(1 \mathrm{H}, \mathrm{d}, 8.0)$ & 116.88 \\
\hline $4^{\prime}$ & - & 149.66 & - & 161.30 \\
\hline $5^{\prime}$ & $6.81(1 \mathrm{H}, \mathrm{d}, 8.0)$ & 116.58 & $6.84(1 \mathrm{H}, \mathrm{d}, 8.0)$ & 116.88 \\
\hline $6^{\prime}$ & $6.99(1 \mathrm{H}, \mathrm{dd}, 2.0,8.0)$ & 123.13 & $7.51(1 \mathrm{H}, \mathrm{d}, 8.0)$ & 131.30 \\
\hline $7^{\prime}$ & $7.61(1 \mathrm{H}, \mathrm{d}, 15.9)$ & 147.33 & $7.68(1 \mathrm{H}, \mathrm{d}, 15.9)$ & 146.04 \\
\hline $8^{\prime}$ & $6.38(1 \mathrm{H}, \mathrm{d}, 15.9)$ & 115.18 & $6.45(1 \mathrm{H}, \mathrm{d}, 15.9)$ & 115.23 \\
\hline $9^{\prime}$ & - & 168.93 & - & 168.89 \\
\hline \multicolumn{5}{|c|}{ Allose } \\
\hline $1^{\prime \prime}$ & $4.76(1 \mathrm{H}, \mathrm{d}, 8.0)$ & 100.86 & $4.76(1 \mathrm{H}, \mathrm{d}, 8.0)$ & 100.80 \\
\hline $2^{\prime \prime}$ & $3.65(1 \mathrm{H}, \mathrm{m})$ & 73.86 & $3.65(1 \mathrm{H}, \mathrm{m})$ & 73.89 \\
\hline $3^{\prime \prime}$ & $5.75(1 \mathrm{H}, \mathrm{t}, 3.0)$ & 71.22 & $5.75(1 \mathrm{H}, \mathrm{t}, 3.0)$ & 69.95 \\
\hline $4^{\prime \prime}$ & $3.76(1 \mathrm{H}, \mathrm{m})$ & 67.17 & $3.76(1 \mathrm{H}, \mathrm{m})$ & 67.15 \\
\hline $5^{\prime \prime}$ & $3.82(1 \mathrm{H}, \mathrm{m})$ & 76.06 & $3.82(1 \mathrm{H}, \mathrm{m})$ & 76.03 \\
\hline $6^{\prime \prime}$ & $3.69(1 \mathrm{H}, \mathrm{m}) ; 3.92(1 \mathrm{H}, \mathrm{m})$ & 62.74 & $3.70(1 \mathrm{H}, \mathrm{m}) ; 3.92(1 \mathrm{H}, \mathrm{m})$ & 62.71 \\
\hline \multicolumn{5}{|c|}{ Rhamnose } \\
\hline $1^{\prime \prime \prime}$ & $4.94(1 \mathrm{H}, \mathrm{s})$ & 98.49 & $4.95(1 \mathrm{H}, \mathrm{s})$ & 98.44 \\
\hline $2^{\prime \prime \prime}$ & $3.6-3.8(1 \mathrm{H}, \mathrm{m})$ & 72.31 & $3.6-3.8(1 \mathrm{H}, \mathrm{m})$ & 72.25 \\
\hline $3^{\prime \prime \prime}$ & $3.6-3.8(1 \mathrm{H}, \mathrm{m})$ & 72.05 & $3.6-3.8(1 \mathrm{H}, \mathrm{m})$ & 72.02 \\
\hline $4^{\prime \prime \prime}$ & $3.42(1 \mathrm{H}, \mathrm{m})$ & 73.80 & $3.42(1 \mathrm{H}, \mathrm{m})$ & 73.79 \\
\hline $5^{\prime \prime \prime}$ & $4.02(1 \mathrm{H}, \mathrm{m})$ & 69.99 & $4.02(1 \mathrm{H}, \mathrm{m})$ & 71.19 \\
\hline $6^{\prime \prime \prime}$ & $1.25(3 \mathrm{H}, \mathrm{d}, 6.0)$ & 18.07 & $1.26(3 \mathrm{H}, \mathrm{d}, 6.0)$ & 18.02 \\
\hline
\end{tabular}

Table 3. ${ }^{1} \mathrm{H}-\mathrm{NMR}\left(\mathrm{CD}_{3} \mathrm{OD}, 400 \mathrm{MHz}\right)$ and ${ }^{13} \mathrm{C}-\mathrm{NMR}\left(\mathrm{CD}_{3} \mathrm{OD}, 100 \mathrm{MHz}\right)$ data of compounds 5 and 6.

$6.84(2 \mathrm{H}, \mathrm{d}, J=8.0 \mathrm{~Hz})]$ and trans-olefinic protons $[7.68(1 \mathrm{H}, \mathrm{d}, J=15.9 \mathrm{~Hz}), 6.45(1 \mathrm{H}, \mathrm{d}, J=15.9 \mathrm{~Hz})]\}$, which was also supported by the literature ${ }^{25}$. Furthermore, the HMBC correlation of $\mathbf{5}$ and $\mathbf{6}$ (Fig. 3) also showed similar modes of long-range correlations with 1. Therefore, the structures of compounds $\mathbf{5}$ and $\mathbf{6}$ were elucidated as 2-(4-hydroxyphenyl)-ethyl-O-a-L-rhamnopyranosyl-( $1 \rightarrow 2)$-(3-O-trans-caffeoyl)- $\beta$-D-allopyranoside and 2-(3, 4-dihydroxyphenyl)-ethyl-O-a-L-rhamnopyranosyl-( $1 \rightarrow 2)$-(3-O-trans-coumaroyl)- $\beta$-D-allopyranoside, respectively.

The other compounds were identified as magnoloside A (1), magnoloside B (7), magnoloside D (4), and magnoloside $\mathrm{E}(\mathbf{9})$ by comparing their ${ }^{1} \mathrm{H}-\mathrm{NMR}$ and ${ }^{13} \mathrm{C}$ NMR data with those reported in the literature ${ }^{16}$. All compounds were obtained from M. officinalis var. biloba fruits for the first time.

In the last few years, some confusing nomenclatures were found in the original articles ${ }^{36,37}$. For example, compound $\mathbf{2}$ and compound $\mathbf{8}$, mentioned in this article, were both given the same nomenclature as magnoloside $\mathrm{F}$ though they actually posses different structures, while magnoloside $\mathrm{F}^{36}$ and magnoloside $\mathrm{M}^{37}$ were characterized by the same structure (compound 2 , mentioned in this article) but different nomenclature. In this manuscript, we propose a reasonable rule of nomenclature in view of the rich structure type of phenylethanoid glycosides. Thus, the confusion and ambiguity caused by two research groups could be clarified. Different types of phenylethanoid glycosides are numberd with Roman numerals (I, II, III, IV, V...), and isomers with subscripts a, b, c... are distinguished. Therefore, compounds $1,2,4,5,6,7,8$ and 9 were renamed magnoloside $I_{a}$ (old name was magnoloside $\mathrm{A}^{32}$ ), magnoloside $\mathrm{I}_{\mathrm{c}}$ (old names were magnoloside $\mathrm{F}^{31}$ and magnoloside $\mathrm{M}^{37}$ ), magnoloside $\mathrm{I}_{\mathrm{b}}$ (old name was magnoloside $\mathrm{D}^{16}$ ), magnoloside $\mathrm{III}_{\mathrm{a}}$ (old name was magnoloside $\mathrm{H}^{36}$ ), magnoloside $\mathrm{IV}_{\mathrm{a}}$ (old name was magnoloside $\mathrm{G}^{36}$ ), magnoloside $\mathrm{II}_{\mathrm{a}}$ (old name was magnoloside $\mathrm{B}^{33}$ ), magnoloside $\mathrm{II}_{\mathrm{b}}$ (old name was magnoloside $\mathrm{F}^{37}$ ), magnoloside $\mathrm{V}_{\mathrm{a}}$ (old name was magnoloside $\mathrm{E}^{16}$ ).

Free radical scavenging activities and their structure-activity relationship. In vitro DPPH radical scavenging, ABTS radical scavenging, and superoxide anion radical scavenging activities of the isolated 


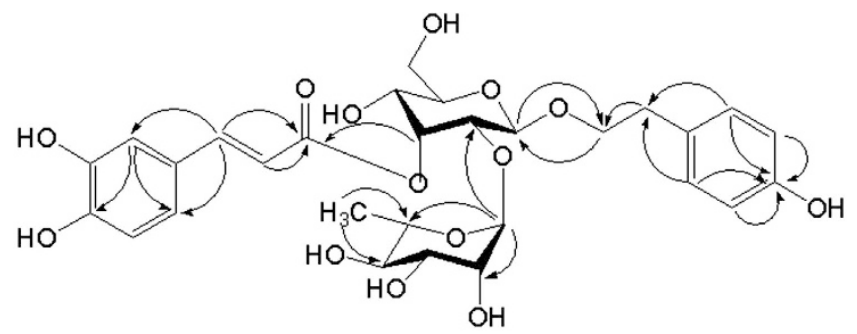

compound 5

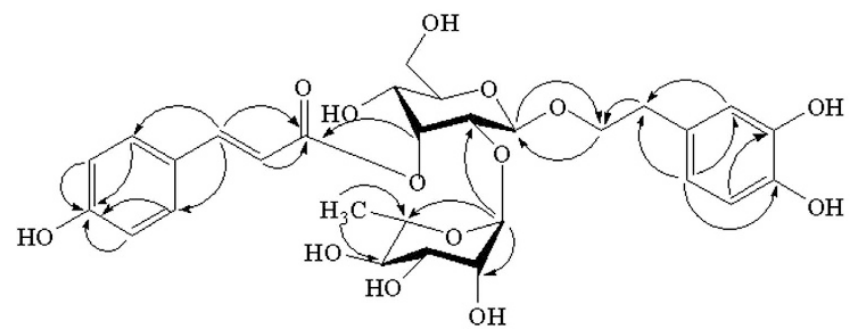

compound 6

Figure 3. Key HMBC correlations $(\mathrm{H} \rightarrow \mathrm{C})$ of compounds 5 and 6 .

\begin{tabular}{|l|c|c|c|}
\hline \multirow{2}{*}{ Compound } & \multicolumn{2}{|c|}{ IC $_{\mathbf{5 0}}(\boldsymbol{\mu M})$} & $\mathbf{K}_{\mathbf{b}}\left(\times \mathbf{1 0}^{-\mathbf{4}} \mathbf{A} / \mathbf{s}\right)$ \\
\cline { 2 - 4 } & $\mathbf{D P P H}$ & ABTS & $\begin{array}{c}\text { Superoxide } \\
\text { anion }\end{array}$ \\
\hline $\mathbf{1}$ & $11.79 \pm 0.57$ & $3.52 \pm 0.11$ & $10.37 \pm 0.33$ \\
\hline $\mathbf{2}$ & $12.99 \pm 0.48$ & $3.53 \pm 0.28$ & $10.68 \pm 0.49$ \\
\hline $\mathbf{3}$ & $21.38 \pm 0.52$ & $3.28 \pm 0.35$ & $11.57 \pm 0.17$ \\
\hline $\mathbf{4}$ & $16.23 \pm 0.16$ & $3.75 \pm 0.11$ & $10.50 \pm 0.18$ \\
\hline $\mathbf{5}$ & $32.18 \pm 0.97$ & $4.16 \pm 0.25$ & $11.22 \pm 0.23$ \\
\hline $\mathbf{6}$ & $35.17 \pm 0.22$ & $5.09 \pm 0.07$ & $9.84 \pm 0.38$ \\
\hline $\mathbf{7}$ & $22.94 \pm 0.26$ & $4.61 \pm 0.10$ & $9.41 \pm 0.77$ \\
\hline $\mathbf{8}$ & $24.62 \pm 0.15$ & $4.78 \pm 0.08$ & $9.07 \pm 0.76$ \\
\hline $\mathbf{9}$ & $20.99 \pm 0.50$ & $6.23 \pm 0.06$ & $8.69 \pm 0.70$ \\
\hline Vc & $40.94 \pm 0.78$ & $11.51 \pm 1.05$ & $0.09 \pm 0.02$ \\
\hline BHT & $89.94 \pm 4.57$ & $7.29 \pm 0.33$ & $10.40 \pm 0.55$ \\
\hline control & - & - & $15.35 \pm 0.19$ \\
\hline
\end{tabular}

Table 4. Radical scavenging activities of phenylethanoid glycosides from M. officinalis var. biloba fruits. Values were the means $\pm \mathrm{SD}, \mathrm{n}=3$; Vc is ascorbic acid; BHT is butylated hydroxytoluene.

phenylethanoid glycosides are summarized in Table 4. The table illustrates that all the isolated phenylethanoid glycosides showed excellent free radical scavenging activity. The analysis of the structure-activity relationship of these phenylethanoid glycosides in the free radical scavenging activity assay suggested that the presence of two adjacent phenolic groups in the molecule resulted in strong free radical scavenging activity. The more two adjacent phenolic groups were, the stronger the free radical scavenging activity was. Meanwhile, all isolated phenylethanoid glycosides were cinnamic acid derivatives, which contain $\alpha, \beta$-conjugated unsaturated ester structures, thereby increasing benzene ring plane conjugation and allowing electron delocalization to stabilize free radicals. This conclusion was well supported by previous reports ${ }^{38,39}$.

In the DPPH radical scavenging assay, compound $\mathbf{1}$ and its structural analogs $(\mathbf{2}, \mathbf{4}$, and $\mathbf{9})$ showed significant DPPH radical scavenging activity with far smaller $\mathrm{IC}_{50}$ values $(11.79 \pm 0.57,12.99 \pm 0.48,16.23 \pm 0.16$, and $20.99 \pm 0.50 \mu \mathrm{M}$, respectively) than the positive controls, such as $\mathrm{V}_{\mathrm{C}}(40.94 \pm 0.78 \mu \mathrm{M})$ and BHT $(89.94 \pm 4.57 \mu$ $\mathrm{M})$. Compounds 3, 7, and $\mathbf{8}$ expressed inferior activity compared with the above four compounds because their $\mathrm{IC}_{50}$ values $\left(21.38 \pm 0.52,22.94 \pm 0.26\right.$, and $24.62 \pm 0.15 \mu \mathrm{M}$, respectively) were near the value of $\mathrm{V}_{\mathrm{C}}$. Moreover, Compound 5 and its structural isomer 6 showed large $\mathrm{IC}_{50}$ values $(32.18 \pm 0.97$ and $35.17 \pm 0.22 \mu \mathrm{M}$, respectively). Some observations could be made according to the above results. Compounds 3,7 , and $\mathbf{8}$ possessed larger steric hindrance because they contained three sugars, whereas compounds $\mathbf{1}, \mathbf{2}, \mathbf{4}$, and 9 only contained two sugars. The increased steric hindrance effect of compounds 3,7 , and $\mathbf{8}$ prevented them from easily approaching the free radicals, so their DPPH radical scavenging capacity was relatively weak than compounds $\mathbf{1 , 2 , 4}$, and $\mathbf{9}$. 

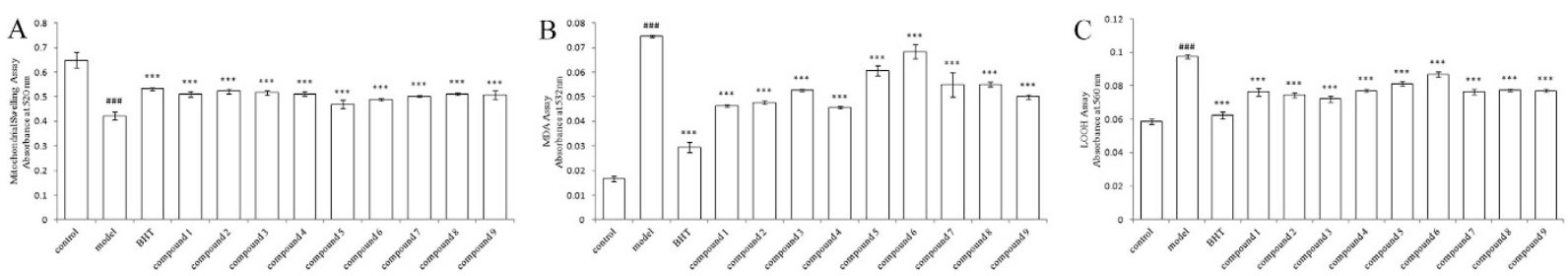

Figure 4. Protective activity of nine compounds against UVB-induced formation of mitochondrial swelling degree (A), MDA (B), and LOOH (C) in rat liver mitochondria. Data are presented as the mean $\pm \operatorname{SD}(n=3)$. Symbols represent statistical significance. ${ }^{* *} p<0.001$, compared with the model group; ${ }^{\# \#} p<0.001$, compared with the control group. BHT is butylated hydroxytoluene.

Moreover, compared with the other seven compounds, compounds 5 and $\mathbf{6}$ belonged to phenylethanoid glycosides with two adjacent phenolic groups only in one side, so they exhibited poor activity.

The ABTS radical scavenging assay demonstrated that compounds $\mathbf{1}, \mathbf{2}$, and $\mathbf{4}$ also exhibited good ABTS radical scavenging activity, but the $\mathrm{IC}_{50}$ value $(6.23 \pm 0.06 \mu \mathrm{M})$ of compound 9 was large. This result was different from that of the DPPH radical scavenging assay, indicating that the apiose group in compound 9 produced a negative effect on the ABTS radical scavenging assay. By contrast, the ABTS radical scavenging ability of compound $\mathbf{3}$ was far better than that of compounds 7 and $\mathbf{8}$, which could be due to the glucose group in compound $\mathbf{3}$. The superoxide anion radical scavenging assay showed that the activity of compound 9 was the best, whereas the activity of compound 3 was the worst. These completely opposite results may be due to the fact that the apiose group in compound 9 enhanced the influence on the superoxide anion radical scavenging assay, whereas the glucose group in compound 3 minimized this influence. These different results obtained from three assays may be explained by the various mechanisms of these assays, suggesting that combined assay methods should be adopted in the screening and evaluation of bioactive compounds from natural materials.

Our experimental results also showed that the substitution position of the caffeoyl group also influenced free radical scavenging activity. By analyzing the structure of similar compounds and experimental results, we found that the free radical scavenging activity of 3-caffeoyl substitution was optimal, followed by 4-caffeoyl substitution and 6-caffeoyl substitution. This conclusion could be supported by the order of activity as follows: $1>\mathbf{2}>\mathbf{4}, \mathbf{7}>\mathbf{8}$.

Protective effects against free radical-induced oxidative damage. Repeated ultraviolet $\mathrm{B}$ (UVB) exposure or constant treated with $\mathrm{Fe}^{2+} / \mathrm{H}_{2} \mathrm{O}_{2}$ can produce reactive oxygen species (ROS), which lead to various adverse effects on the body tissues ${ }^{40,41}$. The free radicals could attack on the fatty acid component of membrane lipids, and then resulting in mitochondrial damage and lipid peroxidation. These facts have been previously demonstrated in mitochondria ${ }^{42}$. Therefore, UVB-induced oxidative damage model and $\mathrm{Fe}^{2+} / \mathrm{H}_{2} \mathrm{O}_{2}$-induced oxidative damage model are classical mitochondria models which are used to measure the protective effect against free radical-induced oxidative damage.

When mitochondria are damaged, swelling will occur; thus, the value of $\mathrm{A}_{520}$ will be reduced ${ }^{43}$. Malondialdehyde (MDA) and lipid hydroperoxide (LOOH) are two relatively unstable products of lipid peroxidation, which is a process where ROS degrade polyunsaturated fatty acids ${ }^{41}$. These toxic products could cause toxic stress in mitochondria, accelerate further oxidative damage ${ }^{44}$. Antioxidant enzymes, such as catalase (CAT), glutathione reductase (GR), and superoxide dismutase (SOD) can confer protection against oxidative stress and tissue damage. These enzymes are critical for defense against the harmful effects of ROS and free radicals in biological systems ${ }^{45}$. In this study, compounds 1-9 were investigated for their protective effects against free radical-induced oxidative damage.

In mitochondrial damage model caused by UVB (Fig. 4), the model group showed a significant decrease in mitochondrial swelling assay, and the change was significantly reversed during treatment with test compounds $(p<0.001)$. The $\mathrm{A}_{532}$ value and $\mathrm{A}_{560}$ value of the MDA $(0.075 \pm 0.001)$ and LOOH $(0.097 \pm 0.001)$ were significantly increased in the model group compared with the control group $(p<0.001)$. However, the test compound groups reduced the MDA and LOOH level and showed significant effects $(p<0.001)$. In mitochondrial damage model caused by $\mathrm{Fe}^{2+} / \mathrm{H}_{2} \mathrm{O}_{2}$, compared with the control group, the model group showed a significant decrease in CAT, GR and SOD levels, together with a significant increase in the level of MDA and LOOH (Fig. 5). Overall, these changes were significantly reversed during treatment with test compounds. However, the level of improvement of some group was not significant $(p>0.05)$.

Comparing the above experimental results, we found that protective effect of compounds containing two pair of two adjacent phenolic groups was optimal, followed by compounds containing one pair of two adjacent phenolic groups (such as compounds 5 and 6). Furthermore, compounds with two sugars were best. These results were consistent with the experimental results of free radical scavenging assays. Therefore, we could speculate that the protective effects of the nine phenylethanoid glycosides against free radical-induced oxidative damage were attributed to their radical scavenging activity, which was caused by the number of two adjacent phenolic groups.

Potential of $M$. officinalis var. biloba fruits as a promising functional alternative food resource. Oral malodor is a major social and psychological problem that affects the majority of the general population ${ }^{20}$. Oral malodor is divided into two kinds, namely, pathological and physiological. Pathological oral malodor is mainly caused by oral diseases, such as caries. Physiological oral malodor is the result of lipid peroxidation from gastrointestinal food debris. Studies ${ }^{2,4,20}$ have proven that the antioxidant and antimicrobial activities 

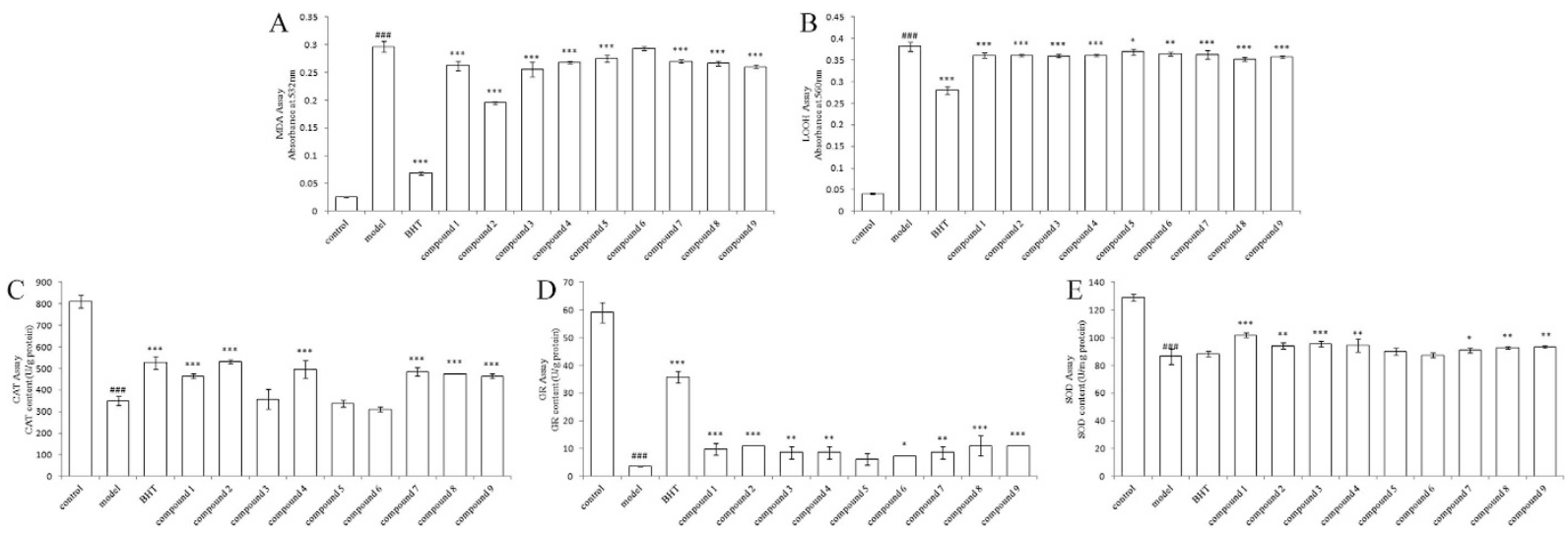

Figure 5. Protective activity of nine compounds against $\mathrm{Fe}^{2+} / \mathrm{H}_{2} \mathrm{O}_{2}$-induced formation of MDA (A), LOOH (B), CAT (C), GR (D) and SOD (E) in rat liver mitochondria. Data are presented as the mean $\pm \operatorname{SD}(n=3)$. Symbols represent statistical significance. ${ }^{* *} p<0.001$, compared with the model group; ${ }^{* *} p<0.01$, compared with the model group; ${ }^{\star} p<0.05$, compared with the model group; ${ }^{\# \#} p<0.001$, compared with the control group. BHT is butylated hydroxytoluene.

of magnolol and honokiol are responsible for oral malodor combat. In the present study, phenylethanoid glycosides were demonstrated to have free radical scavenging activities and inhibitory effects of lipid peroxidation. Thus, phenylethanoid glycosides could assist in improving the condition of physiological oral malodor. $M$. officinalis var. biloba fruits should be used as an alternative food resource added in gum and mints. More studies are necessary to further understand the action mechanism of phenylethanoid glycosides. These studies should also add further value to this by-product of $M$. officinalis var. biloba fruits.

\section{Methods}

Plant material. M. officinalis var. biloba fruits were collected from the mountain area in Enshi (N: $30^{\circ} 17^{\prime}$ 51.56", E: 109²8'27.33"; A: 1,545 m), Hubei Province, China, and identified by Prof. Youwei Wang (Wuhan University, Wuhan, China). A voucher specimen (No. 14610) was deposited at the Traditional Chinese Medicine Specimen Museum, School of Pharmaceutical Science, Wuhan University, China.

Chemical and reagents. 2,2'-Azinobis (3-ethylbenzthiazoline-6-sulphonic acid) (ABTS) was procured from Fluka (Menlo Park, CA, USA). 2,2-Diphenyl-1-picrylhydrazyl (DPPH), high-performance liquid chromatography (HPLC)-grade methanol, and acetonitrile were purchased from Sigma-Aldrich Co. (St. Louis, USA). D-Allose (D1218014), L-rhamnose (B1504021), L-cysteine methyl ester hydrochlorides (G14118068), and 2-thiobarbituric acid (C1510117) were purchased from Aladdin Industrial Corporation (Shanghai, China). Diagnostic kits for CAT, GR, and SOD were purchased from Nanjing Jiancheng Technology Co. Ltd. (Nanjing, China). Ascorbic acid (Vc) (F20060609), ammonium iron (II) sulfate hexahydrate (20120215), butylated hydroxytoluene (BHT) (20121211), D-glucose (20140724), dodecyl sodium sulfate (20130625), hydrogen peroxide 30\% (20150911), iron (II) sulfate heptahydrate (20100506), methanol- $d_{4}$ (20140519), xylenol orange (20100826), and other analytical chemicals and reagents were purchased from Sinopharm Chemical Regent Co. Ltd. (Shanghai, China).

Instrumentation. All NMR spectra $\left({ }^{1} \mathrm{H} \mathrm{NMR}^{13}, \mathrm{C} \mathrm{NMR}, \mathrm{H}-\mathrm{H}\right.$ COSY, HMQC, and HMBC spectra) were obtained on a Bruker DPX-400 spectrometer using standard Bruker pulse programs (Bruker BioSpin GmbH, Rheinstetten, Germany) with solvent peak as references. HR-ESI-MS data were obtained on a Bruker Daltonic micrOTOF-Q II MS spectrometer (Bruker BioSpin GmbH, Rheinstetten, Germany). UV absorption spectra were recorded on a UV-2600 ultraviolet and visible spectrophotometer (Shimadzu, Tokyo, Japan) from $200 \mathrm{~nm}$ to $900 \mathrm{~nm}$. Infrared (IR) spectra were recorded on a Nicolet Nexus 470 spectrometer (Nicolet, Madison, American) using the compounds in the KBr pellet form. Preparative HPLC was carried out on a LabAllance Model 201 HPLC system (LabAllance, Tianjin, China) and a Kromasil C18 column preparative column $(10 \mu \mathrm{m}$, $250 \times 30 \mathrm{~mm}$, Kromasil, Stockholm, Sweden).

Preparation of plant extracts. The dried and powdered M. officinalis var. biloba fruits (5.1 kg, size less than $0.25 \mathrm{~mm}$ ) were extracted three times using 10 volumes of $70 \%$ alcohol $(50 \mathrm{~L})$ for $3 \mathrm{~h}$ at reflux. The filtrate was combined and concentrated under vacuum to yield a dried alcohol extract $(804.9 \mathrm{~g})$. The alcohol extract was suspended in distilled water $(10 \mathrm{~L})$ and then successively partitioned three times with chloroform $(20 \mathrm{~L})$, ethyl acetate $(10 \mathrm{~L})$, and $n$-butanol alcohol $(10 \mathrm{~L})$, which yielded the chloroform fraction $(398.4 \mathrm{~g})$, ethyl acetate fraction $(180.8 \mathrm{~g})$, and $n$-butanol fraction $(63.4 \mathrm{~g})$, respectively. All fractions were stored at $-20^{\circ} \mathrm{C}$ until further use.

Isolation and purification procedures of phenylethanoid glycosides. The $n$-butanol fraction (20g) prepared from the previously described steps was further isolated on a preparative HPLC system. Each chromatographic run was carried out at a flow rate of $20 \mathrm{~mL} / \mathrm{min}$ with a binary mobile phase consisting of methanol (A) 
and $0.1 \%$ formic acid (B) using a step gradient profile. The gradient started with $10 \% \mathrm{~A}$, was varied to $35 \% \mathrm{~A}$ at $10 \mathrm{~min}, 46 \% \mathrm{~A}$ at $40 \mathrm{~min}, 48 \% \mathrm{~A}$ at $43 \mathrm{~min}, 100 \% \mathrm{~A}$ at $50 \mathrm{~min}$, and $100 \% \mathrm{~A}$ isocratic for $10 \mathrm{~min}$, and decreased to $10 \% \mathrm{~A}$ in $0.1 \mathrm{~min}$. After re-equilibration at $10 \% \mathrm{~A}$ for $12 \mathrm{~min}$, the next sample was injected. The temperature of the column oven was $25^{\circ} \mathrm{C}$, and $100 \mu \mathrm{L}(300 \mathrm{mg} / \mathrm{mL})$ was injected into the system every time. The peaks adsorbed at $315 \mathrm{~nm}$ were recorded. Compounds $1(797.7 \mathrm{mg}), 2(226.4 \mathrm{mg}), 3(195.9 \mathrm{mg})$, and $4(298.3 \mathrm{mg})$ were obtained at the retention times of $22.4,32.6,35.3$, and $41.7 \mathrm{~min}$, respectively. The subfraction that was collected at a retention time from $27 \mathrm{~min}$ to $29 \mathrm{~min}$ was further applied to the preparative HPLC system, which was eluted isocratically with $18 \%$ acetonitrile in water (containing $0.1 \%$ formic acid) at a flow rate of $20 \mathrm{~mL} / \mathrm{min}$. Compounds $5(56.0 \mathrm{mg})$ and $6(34.1 \mathrm{mg})$ were then purified. Simultaneously, the subfraction obtained at a retention time of $19.3 \mathrm{~min}$ was subjected to the preparative HPLC system, which was eluted isocratically with $26 \%$ methanol in water at a flow rate of $20 \mathrm{~mL} / \mathrm{min}$. Compounds $7(37.7 \mathrm{mg})$ and $\mathbf{8}(52.7 \mathrm{mg})$ were then obtained. The subfraction obtained at a retention time of $38.4 \mathrm{~min}$ was further isolated over the preparative HPLC system, which was eluted isocratically with $43 \%$ methanol in water at a flow rate of $20 \mathrm{~mL} / \mathrm{min}$ to yield compound $\mathbf{9}(35.9 \mathrm{mg})$.

Acid hydrolysis and sugar analysis in glycosides. Sugar analysis of compounds 2, 3, 5, 6 and 8 was carried out according to the method described by previous studies ${ }^{46,47}$. In brief, compounds $\mathbf{2 , 3 , 5 , 6}$ and 8 (each $4.0 \mathrm{mg}$ ) were hydrolyzed by heating in $4 \mathrm{M} \mathrm{CF}_{3} \mathrm{COOH}(4 \mathrm{~mL})$ at $95^{\circ} \mathrm{C}$ for $4 \mathrm{~h}$. After cooling, the solution was extracted with $\mathrm{CH}_{2} \mathrm{Cl}_{2}(3 \times 2 \mathrm{~mL})$. The water layer was concentrated by reducing pressure and dried by vacuum. Anhydrous pyridine $(0.5 \mathrm{~mL})$ and L-cysteine methyl ester hydrochloride $(2 \mathrm{mg})$ were added and reacted at $60^{\circ} \mathrm{C}$ for $1 \mathrm{~h}$. After cooling to room temperature, o-tolyl isothiocyanate $(10 \mu \mathrm{L})$ was added to the mixture and further heated at $60^{\circ} \mathrm{C}$ for $1 \mathrm{~h}$. The reaction mixture was directly analyzed by reversed-phase HPLC using a Diamonsil C18 analytical column $(5 \mu \mathrm{m}, 250 \times 4.6 \mathrm{~mm})$, which was eluted isocratically with $25 \%$ acetonitrile in water (containing $0.1 \%$ formic acid) at a flow rate of $1 \mathrm{~mL} / \mathrm{min}$. The temperature of the column oven was $25^{\circ} \mathrm{C}$, and $20 \mu$ $\mathrm{L}$ was injected into the system every time. The UV spectra were collected at $250 \mathrm{~nm}$. Peaks of the hydrolysate of compounds $2,3,5,6$ and $\mathbf{8}$ were identified by comparing the retention times of authentic samples of D-allose $\left(t_{R}=19.330\right)$, D-glucose $\left(t_{R}=18.566\right)$, and L-rhamnose $\left(t_{R}=33.411\right)$ after simultaneous treatment under the same conditions.

DPPH and ABTS radical scavenging assays. DPPH and ABTS, two relatively stable free radical compounds widely used to test free radical scavenging activity, were determined using a previously described method $^{48,49}$. Radical scavenging activity was calculated using the equation: radical scavenging activity $(\%)=\left[\left(\mathrm{A}_{0}\right.\right.$ $\left.-\mathrm{A}) / \mathrm{A}_{0}\right] \times 100$ (where $\mathrm{A}_{0}$ is the absorbance of the control, and $\mathrm{A}$ is the absorbance of the test sample). $\mathrm{V}_{\mathrm{C}}$ and BHT with the same concentrations as the samples were used as positive controls.

Superoxide anion radical $\left(\mathrm{O}_{2}^{-}\right)$scavenging assay. The superoxide anion radical is the most common free radical generated in vivo. The capacity of each compound to scavenge superoxide radicals was examined by a pyrogallol auto-oxidation system ${ }^{50}$. Pyrogallic acid can auto-oxidize under alkaline conditions to produce $\mathrm{O}_{2}{ }^{-}$ directly, and the rate constant of this auto-oxidation reaction is dependent on the $\mathrm{O}_{2}{ }^{-}$concentration. If the test compound can scavenge $\mathrm{O}_{2}^{-}$, the auto-oxidation reaction can slow down significantly. The auto-oxidation rate constant $\left(\mathrm{K}_{\mathrm{b}}\right)$ of pyrogallic acid was calculated from the curve of $\mathrm{A}_{325} \mathrm{~nm}$ versus time. $\mathrm{V}_{\mathrm{C}}$ and BHT were used for comparative purposes. Simultaneously, the $\mathrm{K}_{\mathrm{b}}$ value $\left(10^{-4} \mathrm{~A} / \mathrm{s}\right)$ of the blank control was recorded.

Test animals. Sprague-Dawley (SD) rats $(200 \pm 20 \mathrm{~g})$ were purchased from the Laboratory Animal Center of Wuhan University, Wuhan, China. All the rats were housed under regulated conditions of $12 \mathrm{~h}$ light/12 h dark cycles, $25 \pm 2{ }^{\circ} \mathrm{C}$, and $30 \%-60 \%$ relative humidity. They were fed with a standard pellet diet (rat feed, purchased from the Laboratory Animal Center of Wuhan University, Wuhan, China) and unlimited water. The animals were allowed to acclimatize to the room conditions for 2 days. All experimental procedures were approved by the Institutional Animal Ethical Committee of the Committee for the Purpose of Control and Supervision of Experiments on Animals, Wuhan University in Wuhan, China. The experimental methods were performed in accordance with "Regulations for the Administration of Affairs Concerning Experimental Animals" and "Guiding Opinions of Treating Experimental Animals with Good Ethics", which have been formally released and implemented by Chinese Government.

Preparation of mitochondria. Mitochondria were isolated from SD rats according to the method described by previous studies ${ }^{45}$. All procedures were carried out at $4{ }^{\circ} \mathrm{C}$.

Mitochondrial damage model caused by UVB. The experiment consisted of the control group, model group, BHT group, and test sample group. Each group comprised the following set up: for the control group, $0.5 \mathrm{~mL}$ of mitochondrial protein $+0.1 \mathrm{~mL}$ of phosphate buffer $(0.05 \mathrm{M}, \mathrm{pH} 7.4)$; for the model group, $0.5 \mathrm{~mL}$ of mitochondrial protein $+0.1 \mathrm{~mL}$ of phosphate buffer $(0.05 \mathrm{M}, \mathrm{pH} 7.4)+\mathrm{UVB}$; for the BHT group, $0.5 \mathrm{~mL}$ of mitochondrial protein $+0.1 \mathrm{~mL}$ of BHT $(12.5 \mu \mathrm{M}$, in phosphate buffer $)+\mathrm{UVB}$; and for the test compound group, $0.5 \mathrm{~mL}$ of mitochondrial protein $+0.1 \mathrm{~mL}$ of test sample $(12.5 \mu \mathrm{M}$, in phosphate buffer $)+\mathrm{UVB}$. The model, BHT, and test compound groups were irradiated for $4 \mathrm{~h}$ with a $20 \mathrm{~W}$ UVB lamp (TL/12RS, Philips) from a $15 \mathrm{~cm}$ distance. The control group was kept at $37^{\circ} \mathrm{C}$ for $4 \mathrm{~h}$ without irradiation.

Mitochondrial damage model caused by $\mathrm{Fe}^{2+} / \mathrm{H}_{2} \mathrm{O}_{2}$. The experiment consisted of the control group, model group, BHT group, and test sample group. Each group comprised the following set up: for the control group, $0.5 \mathrm{~mL}$ of mitochondrial protein $+0.5 \mathrm{~mL}$ of phosphate buffer $(0.05 \mathrm{M}, \mathrm{pH} 7.4)$; for the model group, $0.5 \mathrm{~mL}$ of mitochondrial protein $+0.5 \mathrm{~mL}$ of phosphate buffer $(0.05 \mathrm{M}, \mathrm{pH} 7.4)+30 \mu \mathrm{L} \mathrm{FeSO}_{4}(10 \mathrm{mM})+20 \mu$ $\mathrm{L} \mathrm{H}_{2} \mathrm{O}_{2}(65 \mathrm{mM})$; for the BHT group, $0.5 \mathrm{~mL}$ of mitochondrial protein $+0.5 \mathrm{~mL}$ of BHT $(12.5 \mu \mathrm{M}$, in phosphate 
buffer $)+30 \mu \mathrm{FeSO}_{4}(10 \mathrm{mM})+20 \mu \mathrm{L} \mathrm{H}_{2} \mathrm{O}_{2}(65 \mathrm{mM})$; and for the test compound group, $0.5 \mathrm{~mL}$ of mitochondrial protein $+0.1 \mathrm{~mL}$ of test sample $(12.5 \mu \mathrm{M}$, in phosphate buffer $)+30 \mu \mathrm{L} \mathrm{FeSO}_{4}(10 \mathrm{mM})+20 \mu \mathrm{L} \mathrm{H}_{2} \mathrm{O}_{2}(65 \mathrm{mM})$. All experiment groups were kept at $37^{\circ} \mathrm{C}$ for $1 \mathrm{~h}$.

Measurement of biochemical indicators. The values of $\mathrm{A}_{520}$ were used to evaluate the mitochondrial swelling degree ${ }^{43}$. MDA assay was evaluated using the thiobarbituric acid method ${ }^{45}$. LOOH assay was measured using a modified version of a previously reported method ${ }^{45}$. CAT, GR and SOD activities in mitochondrial protein were measured by commercial kits purchased from Nanjing Jiancheng Technology Co. Ltd. (Nanjing, China).

Statistical analysis. All experiments were carried out in triplicate, and the results were reported as the mean \pm standard deviation $(n=3)$. The data were analyzed using one-way ANOVA. Statistically significant effects were analyzed, and the means were also compared using least-significant difference (LSD) test. Statistical significance was determined at $p<0.05$.

\section{References}

1. Chinese Pharmacopoeia Commission. Pharmacopoeia of the People's Republic China, 2015 (China Medical Science Press: Beijing, China), 251-252 (2015).

2. Greenberg, M., Dodds, M. \& Tian, M. Naturally occurring phenolic antibacterial compounds show effectiveness against oral bacteria by a quantitative structure-activity relationship study. J. Agric. Food. Chem. 56, 11151-11156 (2008).

3. Tang, S. Y. et al. Characterization of antioxidant and antiglycation properties and isolation of active ingredients from traditional chinese medicines. Free Radical Bio. Med. 36, 1575-1587 (2001).

4. Zhao, C. \& Liu, Z. Q. Comparison of antioxidant abilities of magnolol and honokiol to scavenge radicals and to protect DNA. Biochimie 93, 1755-1760 (2011).

5. Chao, L. K. et al. Anti-inflammatory bioactivities of honokiol through inhibition of protein kinase C, mitogen-activated protein kinase, and the NF-kappaB pathway to reduce LPS-induced TNF alpha and NO expression. J. Agric. Food. Chem. 58, 3472-3428 (2010).

6. Kuo, W. L., Chung, C. Y., Hwang, T. L. \& Chen, J. J. Biphenyl-type neolignans from Magnolia officinalis and their anti-inflammatory activities. Phytochemistry 85, 153-160 (2013).

7. Maruyama, Y., Kuribara, H., Morita, M., Yuzurihara, M. \& Weintraub, S. T. Identification of magnolol and honokiol as anxiolytic agents in extracts of saiboku-to, an oriental herbal medicine. J. Nat. Prod. 61, 135-138 (1998).

8. Watanabe, K. Pharmacology of magnolia bark with special reference to gastrointestinal functions. Gendai Toyo Igaku 7, 54-59 (1986).

9. Liu, Y. et al. The natural compound magnolos inhibits invasion and exhibits potential in hunman breast cancer therapy. Scientific Reports 3, 3098-3105 (2013).

10. Lin, Y. R., Chen, H. H., Ko, C. H. \& Chan, M. H. Neuroprotective activity of honokiol and magnolol in cerebellar granule cell damage. Eur. J. Pharmacol. 537, 64-69 (2006).

11. Jennifer, H., Ho, C. \& Hong, C. Y. Cardiovascular protection of magnolol: cell-type specificity and dose-related effects. J. Biomed. Sci. 19, 70-78 (2012).

12. Zhang, B. B., Wang, H. \& Chen, S. Z. Quantification and structural identification of related phenolic compounds in the raw medicinal material honokiol. J.Chi.Pharm. Sci. 22, 420-426 (2013).

13. Yahara, S., Nishiyori, T., Kohda, A., Nohara, T. \& Nishioka, I. Isolation and charaterization of phenolic compounds from Magnoliae Cortex produced in China. Chem. Pharm. Bull. 39, 2024-2036 (1991).

14. Yan, R. Y., Liu, H. L., Zhang, J. Y. \& Yang B. Phenolic glycosides and other constituents from the bark of Magnolia officinalis. J. Asian Nat. Prod. Res. 16, 400-405 (2014).

15. Guo, Z. F. et al. A novel aporphine alkaloid from Magnolia officinalis. Fitoterapia 82, 637-641 (2011).

16. Yu, S. X., Yan, R. Y., Liang, R. X., Wang, W. \& Yang, B. Bioactive polar compounds from stem bark of Magnolia officinalis. Fitoterapia 83, 356-361 (2012).

17. National health and family planning commission of the people's republic of China. The Ministry of Health informed about further standardize health food raw materials management $\mathbf{5 1}$ (2002).

18. College ter Beoordeling van Geneesmiddelen. Magnolia bark extract http://www.cbg-meb.nl/documenten/rapporten/2010/09/25/ nv-extract-van-magnoliabast (2010).

19. Advisory Committee on Novel Foods and Processes (ACNFP) Secretariat Food Standards Agency. Initial opinion: Magnolia bark supercritical carbon dioxide extract as a food ingredient. Magnolia bark extract. EC No. 107 http://acnfp.food.gov.uk/assess/ fullapplics/magnoliabarkextract (2011).

20. Greenberg, M., Urnezis, P. \& Tian, M. Compressed mints and chewing gum containing magnolia bark extract are effective against bacteria responsible for oral malodor. J. Agric. Food Chem. 55, 9465-9469 (2007).

21. He, J. S. et al. Population structure and genetic diversity distribution in wild and cultivated populations of the traditional Chinese medicinal plant Magnolia officinalis subsp. biloba (Magnoliaceae). Genetica 135, 233-243 (2009).

22. Wang, Y. W. et al. New phenylethanoid glycosides from Magnolia officinalis fruits. Pacifichem 2015, International Chemical Congress of Pacific Basin Societies(Hawaii, USA) 2082 (2015).

23. Li, L. et al. Isolation and purification of phenylethanoid glycosides from Cistanche deserticola by high-speed counter-current chromatography. Food Chem. 108, 702-710 (2008).

24. Nan, Z. D. et al. Phenylethanoid glycosides with anti-inflammatory activities from the stems of Cistanche deserticola cultured in Tarim desert. Fitoterapia 89, 167-174 (2013).

25. Morikawa, T. et al. Acylated phenylethanoid oligoglycosides with hepatoprotective activity from the desert plant Cistanche tubulosa. Bioorg. Med. Chem. 18, 1882-1890 (2010).

26. Song, X. et al. Immunomodulatory effects of crude phenylethanoid glycosides from Ligustrum purpurascens. J. Ethnopharmacol. 144, 584-591 (2010).

27. Liu, Y. L. et al. Antimicrobial, anti-inflammatory activities and toxicology of phenylethanoid glycosides from Monochasma savatieri Franch. ex Maxim. J. Ethnopharmacol. 149, 431-437 (2013).

28. Bardakci, H. et al. Antioxidant activities of several Scutellaria taxa and bioactive phytoconstituents from Scutellaria hastifolia L. Ind. Crop. Prod. 77, 196-203 (2015).

29. Marino, S. D. et al. Antioxidant activity of phenolic and phenylethanoid glycosides from Teucrium polium L. Food Chem. 133, 21-28 (2012).

30. Liu, Y. G. et al. Synthetic phenylethanoid glycoside derivatives as potent neuroprotective agents. Eur. J. Med. Chem. 95, 313-323 (2015).

31. Oyourou, J. N., Combrinck, S., Regnier, T. \& Marston, A. Purification, stability and antifungal activity of verbascoside from Lippia javanica and Lantana camara leaf extracts. Ind. Crop. Prod. 43, 820-826 (2013). 
32. Hasegawa, T., Fukuyama, Y., Yamada, T. \& Nakagawa, K. Isolation and structure of magnoloside A, a new phenylpropanoid glycosides from Magnolia obovata Thunb. Chem. Lett. 17, 163-166 (1988).

33. Hasegawa, T., Fukuyama, Y., Yamada, T. \& Nakagawa, K. Structures of magnoloside B and C. Novel phenylpropanoid glycosides with allopyranose as core the sugar unit. Chem. Pharm. Bull. 36, 1245-1248 (1988).

34. Porter, E. A. et al. Phenylethanoid glycosides in tepals of Magnolia salicifoliaand their occurrence in flowers of Magnoliaceae. Phytochemistry 117, 185-193 (2015).

35. Yoshikawa, M. et al. Phenylethanoid oligoglycosides and acylated oligosugars with vasorelaxant activity from Cistanche tubulosa. Bioorg. Med. Chem. 14, 7468-7475 (2006).

36. Seo, K. H. et al. Phenylethanoid glycosides from the fruits of Magnolia obovata, Chem. Nat. Compd+. 51, 660-665 (2015).

37. Xue, Z. Z., Yan, R. Y. \& Yang, B. Phenylethanoid glycosides and phenolic glycosides from stem bark of Magnolia officinalis. Phytochemistry 127, 50-62 (2016)

38. Es-Safi, N. E., Kollmann, A., Khlifi, S. \& Ducrot, P. H. Antioxidative effect of compounds isolated from Globularia alypum L. structure-activity relationship. LWT - Food Sci. Technol. 40, 1246-1252 (2007).

39. Wu, A. Z., Lin, C. Z. \& Zhu, C. C. Progress in structure-activity relationship of phenylethanoid glycosides. Nat. Prod. Res. Dev. 25, 862-865 (2013).

40. Park, H. M. et al. Extract of Punica granatum inhibits skin photoaging induced by UVB irradiation. Int. J. Dermatol. 49, 276-282 (2010).

41. Oboh, G., Akinyemi, A. J. \& Ademiluyi, A. O. Antioxidant and inhibitory effect of red ginger (Zingiber officinale var. Rubra) and white ginger (Zingiber officinale Roscoe) on $\mathrm{Fe}^{2+}$ induced lipid peroxidation in rat brain in vitro. Exp. Toxicol. Pathol. 64, 31-36 (2012).

42. Bhattacharya, S., Kamat, J. P., Bandyopadhyay, S. K. \& Chattopadhyay, S. Comparative inhibitory properties of some Indian medicinal plant extracts against photosensitization-induced lipid damage. Food Chem. 113, 975-979 (2009).

43. Hunter, F. E. et al. Swelling and lysis of rat liver minochondria induced by ferrous ions. J. Biol. Chem. 238, 828-835 (1963).

44. Zhu, L. et al. Coptis chinensis inflorescence extract protection against ultraviolet-B-induced phototoxicity, and HPLC-MS analysis of its chemical composition. Food Chem. Toxicol. 50, 2584-2588 (2012).

45. Huang, B. et al. In vitro and in vivo evaluation of inhibition activity of lotus (Nelumbo nucifera Gaertn.) leaves against ultraviolet B-induced phototoxicity. J. Photoch. Photobio. B 121, 1-5 (2013).

46. Tanana, T., Nakashima, T., Ueda, T., Tomii, K. \& Kouno, I. Facile discrimination of aldose enantiomers by reversed-phase HPLC. Chem. Pharm. Bull. 55, 899-901 (2007).

47. Tian, E. L., Yang, G. Z., Mei, Z. N. \& Chen, Y. Chemical constituents from stems of Glycosmis pentaphylla. Chin. Tradit. Herb. Drugs 45, 1358-1362 (2014).

48. Mawalagedera, S. M. M. R., Ou, Z. Q., McDowell, A. \& Gould, K. L. Effects of boiling and in vitro gastrointestinal digestion on the antioxidant activity of Sonchus oleraceus leaves. Food Funct. 7, 1515-1522 (2016).

49. Cristina, R. C. \& Rudolf, J. Free radical scavenging (antioxidant activity) of natural dissolved organic matter. Mar. Chem. 177, 668-676 (2015).

50. Zhou, G., Chen, Y. X., Liu, S., Yao, X. C. \& Wang, Y. W. In vitro and in vivo hepatoprotective and antioxidant activity of ethanolic extract from Meconopsis integrifolia (Maxim.) Franch. J. Ethnopharmacol. 148, 664-670 (2013).

\section{Acknowledgements}

This work was supported by the Project of the National Twelve-Five Year Research Program of China (2011BAI06B06; 2012BAI29B03), the Commonweal Specialized Research Fund of China Agriculture (201103016), and the Nanjing 321 plan for Bringing in technological leading talents (2013A12011).

\section{Author Contributions}

L.L.G. and Y.W.W. designed research and wrote the manuscript; L.L.G., W.H.Z., G.Z., B.X.M., Q.G.M. and Y.X.C. contributed experimental data and analysis; All authors reviewed the manuscript.

\section{Additional Information}

Supplementary information accompanies this paper at http://www.nature.com/srep

Competing Interests: The authors declare no competing financial interests.

How to cite this article: Ge, L. et al. Nine phenylethanoid glycosides from Magnolia officinalis var. biloba fruits and their protective effects against free radical-induced oxidative damage. Sci. Rep. 7, 45342; doi: 10.1038/ srep45342 (2017).

Publisher's note: Springer Nature remains neutral with regard to jurisdictional claims in published maps and institutional affiliations.

(c) (1) This work is licensed under a Creative Commons Attribution 4.0 International License. The images or other third party material in this article are included in the article's Creative Commons license, unless indicated otherwise in the credit line; if the material is not included under the Creative Commons license, users will need to obtain permission from the license holder to reproduce the material. To view a copy of this license, visit http://creativecommons.org/licenses/by/4.0/

(C) The Author(s) 2017 